\title{
The Concept of Mass-Density in Classical Thermodynamics and the Boltzmann Kinetic Equation for Dilute Gases
}

\author{
S. Kokou Dadzie and Jason M. Reese \\ University of Strathclyde, Department of Mechanical Engineering, \\ Glasgow G1 1XJ, Scotland, United Kingdom \\ kokou.dadzie@strath.ac.uk
}

\begin{abstract}
In this paper we discuss the mass-density of gas media as represented in kinetic theory. It is argued that conventional representations of this variable in gas kinetic theory contradict a macroscopic field variable and thermodynamic property in classical thermodynamics. We show that in cases where mass-density variations exist throughout the medium, introducing the mass-density as a macroscopic field variable leads to a restructuring of the diffusive/convective fluxes and implies some modifications to the hydrodynamic equations describing gas flows and heat transfer. As an illustration, we consider the prediction of mass-density profiles in a simple heat conduction problem between parallel plates maintained at different temperatures.
\end{abstract}

Keywords: Kinetic Theory; Distribution Function; Statistical Physics; Boltzmann Equation; Compressible Fluids; Continuum Mechanics PACS: 02.50.-r; 05.20-y; 06.30.Dr ; 51.10.+y; 51.30.+i

\section{INTRODUCTION}

The kinetic theory of gases uses statistical mechanics and probability theory concepts such as the probability density distribution of molecules. From this probability density, macroscopic field variables such as the thermodynamic properties (mass-density, volume, pressure, temperature etc.) are constructed by considering moments of the probability density. Given simple monatomic gases, the probability density distribution is defined on the phase space that comprises molecule positions and velocities as two independent random variables, then the moments are taken with respect only to the velocity. Therefore, any physical macroscopic densities, in particular the thermodynamic mass-density of the medium, are associated with reduced probabilities in the position subspace.

The way that measurable macroscopic properties are defined within a molecular-based description of a continuum fluid is not straightforward, and some foundational questions and paradoxes can be raised [1,2]. Maxwell himself circumscribed the use of probability distribution functions in the kinetic theory of gases [3]. Meanwhile, difficulties concerning how a physical mass-density can be obtained from the distribution function involved in the derivation of the Boltzmann kinetic equation have led other kinetic theorists to define a coarse-grained distribution function to embody macroscopic features $[4,5]$.

We present here the contradictions that arise in the gas kinetic theory consideration of physical densities, in particular in the derivations of the kinetic equations. We investigate an approach where a complementary microscopic random variable is introduced in order to incorporate a proper field variable representation of the mass-density of the medium.

\section{BACKGROUND}

Physical space is referenced with a fixed inertial frame $\left(X_{1}, X_{2}, X_{3}\right)$, in which exists a gas. We denote a differential element in the position sub-phase space, $d_{X}=d_{X_{1}} d_{X_{2}} d_{X_{3}}$, and a differential element in the velocity sub-phase space, $d_{\xi}=d_{\xi_{1}} d_{\xi_{2}} d_{\xi_{3}}$. Let us define the following two probability densities:

(A) A probability density function $f_{A}(t, X, \xi)$ such that $f_{A}(t, X, \xi) d_{X} d_{\xi}$ represents the probable number of molecules that, at time $t$, have their positions located within $X \pm d_{X}$ and their velocities within the element $\xi \pm d_{\xi}$.

(B) A probability density function $f_{B}(t, X, \xi)$ regarding an arbitrary single gas molecule, such that $f_{B}(t, X, \xi) d_{X} d_{\xi}$ represents the probability that, at time $t$, the velocity of this single molecule is within the element $\xi \pm d_{\xi}$ and the position of this single molecule is within $X \pm d_{X}$. 
The above two probability density functions are evidently two different concepts. In particular, (A) gives a number of molecules, while (B) does not. Derivation of the Boltzmann kinetic equation in monatomic dilute gases can be found in the literature starting with any of the above probability densities. Starting with the one-molecule distribution function defined in (B), a Liouville equation is written [6]

$$
\frac{\partial f_{B}}{\partial t}+(\xi \cdot \nabla) f_{B}+\left(F_{t o t} \cdot \nabla_{\xi}\right) f_{B}=0
$$

where $\nabla=\left(\partial / \partial X_{1}, \partial / \partial X_{2}, \partial / \partial X_{3}\right)$ is the traditional spatial gradient operator and $\nabla_{\xi}$ denotes the similar operator in the velocity space, i.e., $\nabla_{\xi}=\left(\partial / \partial \xi_{1}, \partial / \partial \xi_{2}, \partial / \partial \xi_{3}\right)$. In equation (1) the third term on the left hand side corresponds to the total force exerted on a given arbitrary molecule. This force encompasses both external actions and the potential forces exerted by the surrounding molecules. The route to the gas kinetic equation is concerned with modelling the force term. Instead of maintaining the continuous action of the intermolecular forces, this force component is replaced by discontinuous changes that occur instantly onto the momentum of the single molecule [6]. The resulting equation is the Boltzmann equation in the one-particle phase space, written when ignoring external forces as

$$
\frac{\partial f_{B}}{\partial t}+(\xi \cdot \nabla) f_{B}=I\left(f_{B}, f_{B}\right)
$$

The term on the right hand side that arose from the intermolecular forces is the collision integral, restricted by the assumption that molecules are uncorrelated in both the position and velocity spaces. For hard-sphere molecules, this is written,

$$
I(f, f)=\int\left[f\left(t, X, \xi^{*}\right) f\left(t, X, \xi_{1}^{*}\right)-f(t, X, \xi) f\left(t, X, \xi_{1}\right)\right] \xi_{r} b d_{b} d_{\varepsilon} d_{\xi_{1}},
$$

where $\xi$ and $\xi_{1}$ refer to post-collision velocities of the interacting molecules, $\xi^{*}$ and $\xi_{1}^{*}$ refer to pre-collision velocities, $\xi_{r}=\left|\xi-\xi_{1}\right|$ is the two colliding molecules' relative velocity, $\varepsilon$ is the azimuthal impact angle, $b$ is the distance of closest approach of the undisturbed trajectories in the centre-of-mass frame of reference. We recall that this collision integral is based on the elementary dynamic laws of a collision between two point-mass molecules and that it does not describe any spatial configuration changes during the collisions apart from exchanges of momentum and energy .

Let us define the following quantity:

$$
B_{n}(t, X)=\int f_{B}(t, X, \xi) d_{\xi},
$$

which defines another probability density function in the position sub-space. According to the definition of the distribution function $f_{B}, B_{n}(t, X) d_{X}$ represents the probability of finding a single molecule in the vicinity of position $X$, regardless of its velocity. In kinetic theory, $B_{n}(t, X)$ is conventionally associated with the mass-density of the medium through the following assertion [7]: consider a fixed number $N$ as the total number of gaseous molecules; assume

$$
f_{A}=N f_{B},
$$

and then interpret $\int f_{A} d_{\xi}$ as an average number of molecules per unit of gas volume; hence $B_{n}(t, X)$ (or $N B_{n}(t, X)$ ). An elementary volume of gas is represented by $d_{X}$, and the distribution function $f_{A}$ follows the same equation (2) because $N$ is just a constant. However, this assertion and equation (5) presupposes that the summation over the one-particle distribution functions corresponding to each individual molecule gives the average number of molecules around a given position. This implies that each molecule is statistically independent (this is without referring to the collision integral), which means the true collective nature of the molecules constituting the medium, and the real spatial configurations of the molecules, are disregarded. For example, the position of a given molecule relative to another is ignored.

A second common route to express the Boltzmann kinetic equation is to start the derivation with the distribution function $f_{A}$ directly. Then equation (4) written with $f_{A}$ reads directly as an average number of molecules per unit volume and this is then interpreted as the mass-density of the medium. In this derivation, it is simply assumed that "the variation of a number of molecules in a cell defined by $\left(\xi \pm d_{\xi}\right) \otimes\left(X \pm d_{X}\right)$ is due to collision between molecules" [8], and that collision itself is an operation occurring only in the velocity sub-space. Then,

$$
\frac{\partial f_{A}}{\partial t}+(\xi \cdot \nabla) f_{A}=I\left(f_{A}, f_{A}\right) .
$$

There are some incompatibilities inherent in this formulation. Considering a position $X$ in the gas, there is not a given single molecule but a collection of molecules associated with this point, and in a similar manner the velocity (some 
references use the term "molecules of a kind" [3, 8]). Accordingly, collisions can be regarded as interactions between two groups of molecules. This contrasts with the usual description of the dynamics of a collision as an interaction between two individual molecules coming from two different positions. The concept of many molecules sitting at the same position at the same time can be regarded as problematic in equation (6).

Specifically, a "mass-density" or "physical density" of a gas medium as it is conceived in classical continuum mechanics is a macroscopic thermodynamic property and therefore an average value. It is viewed as an amount of mass divided by the macroscopic volume in which is spread this mass. While this macroscopic volume is made up of empty spaces and real volumes of the molecular objects, it is itself a thermodynamic variable in classical equilibrium thermodynamics. Accordingly, the mass-density, and its associated specific volume, have at first sight no predefined assignment to the mathematical measures or probability density functions.

Expression (4), written either with $f_{A}$ or $f_{B}$, contradicts the macroscopic field variable and thermodynamic properties of the mass-density: first, equation (4) is not a macroscopic average of any microscopic field variable or random variable, it is rather a reduced probability density. Second, an elementary volume of a gas represented by $d_{X}$ within this definition is a frame-dependent quantity, in contrast with a thermodynamic property that should be a frameindependent quantity.

In some more complex derivations of the Boltzmann equation, such as those based on the Liouville equation written for a complete distribution function of a system of a fixed number $N$ molecules, the mass-density appears as a constant. The mass-density of the medium is defined as a normalization factor $N / V$ in front of a distribution function, where $N$ is the total number of molecules in a fixed volume $V$ of a container [9]. This also shows up in the derivation of an equilibrium solution to the Boltzmann equation. That is, the Maxwell-Boltzmann distribution is essentially a distribution in the velocity subspace regardless of mass-density. The Boltzmann H-theorem, associated with the derivation of the Maxwell-Boltzmann distribution as the only equilibrium distribution, is strictly derived only if it is admitted first that the medium is spatially uniform, and that bounding wall effects are neglected [8]. Otherwise, some other derivations have been based on an asymptotic limit analysis that involves $N$ tending to infinity. Even in this case, problems still remain because as the number of molecules tends to infinity the distribution function also tends to infinity (i.e. the number of molecules per unit of physical volume becomes infinite). Then a re-scaling such as equation (5) has to be used [10].

\title{
A MODIFIED KINETIC APPROACH TO GAS MEDIA
}

\section{A New Probability Density Distribution}

Considering an arbitrary molecule, we define the following probability density distribution:

\begin{abstract}
$f(t, X, \xi, v)$ is such that $f(t, X, \xi, v) d_{X} d_{\xi} d_{v}$ is the probability of an arbitrary single molecule to be, at a given time $t$, located in the vicinity of position $X$ with its velocity in the vicinity of velocity $\xi$, while the configuration of its surrounding molecules at that time is readable with a microscopic parameter whose measurable value is around $v$.
\end{abstract}

Variable $v$ takes a positive value so that $v$ tending to zero represents packed gaseous molecules with no separation distances, and $v$ tending to infinity represents an isolated molecule. A dilute gas properly lies between these two limiting cases. This new variable bears information about other molecules, and the cohesive nature of the medium, and therefore completes the one-molecule description. It is assumed to be a random variable, independent of position and velocity variables; it will not be important if continuum macroscopic field variables based on collections of molecules, such as mass-density and pressure, are not considered (i.e. if we are only to describe a single moving molecule). More precisely, if $d$ is on average the distance between a target single molecule and its surrounding molecules, then the geometrical variable $v$ may be given a handleable value of the volume of the sphere of radius $(d / 2)$, i.e $v=(4 \pi / 3)(d / 2)^{3}$.

A total variation in time of the new one-molecule distribution function is given by,

$$
\frac{\delta f}{\delta t}=\frac{\partial f}{\partial t}+\left(\frac{\delta X}{\delta t} \cdot \nabla\right) f+\left(\frac{\delta \xi}{\delta t} \cdot \nabla_{\xi}\right) f+\frac{\delta v}{\delta t} \frac{\partial f}{\partial v},
$$

where $\delta / \delta t$ denotes the total time derivative following microscopic motions. The rate of change of position with time is the velocity of the molecule, so $\delta X / \delta t=\xi$. The rate of change of momentum with time is the sum of forces exerted 
on the molecule, so $\delta \xi / \delta t=F_{\text {ext }}+F_{\text {int }}$, where $F_{\text {ext }}$ denotes external forces such as gravity, $F_{\text {int }}$ denotes internal forces due to other molecules (per unit mass). The last term in equation (7) results from the local change of $v$, i.e. the change in the spatial configuration of the molecular ensemble due to changes in the properties of the medium.

\section{Definition of Macroscopic Field Variables}

We define first the following average quantity:

$$
A_{n}(t, X)=\int_{-\infty}^{+\infty} \int_{0}^{+\infty} f(t, X, \xi, v) d_{v} d \xi
$$

This quantity refers, according to the definition of the distribution function $f(t, X, \xi, v)$, to a reduced probability in the position space, i.e the probability of finding a molecule around $X$ regardless of its velocity and the distribution of the other molecules. This is not therefore a proper thermodynamic mass-density of the medium.

The local mean value, $\bar{Q}(t, X)$, of any property $Q$ can be defined according to classical statistical mechanics by,

$$
\bar{Q}(t, X)=\frac{1}{A_{n}(t, X)} \int_{-\infty}^{+\infty} \int_{0}^{+\infty} Q f(t, X, \xi, v) d_{v} d \xi .
$$

For example, the local average of $v$, i.e. the local mean-free-volume around each gaseous molecule, is given by,

$$
\bar{v}(t, X)=\frac{1}{A_{n}(t, X)} \int_{-\infty}^{+\infty} \int_{0}^{+\infty} v f(t, X, \xi, v) d_{v} d \xi .
$$

From this mean value of the volume around a molecule we can define a mass-density in the vicinity of position $X$ through:

$$
\bar{\rho}(t, X)=\frac{A_{n}(t, X) M}{A_{n}(t, X) \bar{v}(t, X)}=\frac{M}{\bar{v}(t, X)},
$$

where $M$ is the molecular mass. The specific volume is then given by $\bar{v}(t, X) / M$.

Two mean velocities can be defined using two different weighting values. First, a local mean mass-velocity, $U_{m}(t, X)$, is given through

$$
A_{n}(t, X) U_{m}(t, X)=\int_{-\infty}^{+\infty} \int_{0}^{+\infty} \xi f(t, X, \xi, v) d_{v} d \xi
$$

As the molecular mass is constant in single-component media, it has been canceled out in equation (12). According to the definition of the distribution function, this average velocity can be viewed as the average velocity at which molecules are travelling; it is independent of the mass-density of the medium. Using the microscopic free volume as a weighting, a local mean volume-velocity, $U_{v}(t, X)$, can also be defined:

$$
\bar{v}(t, X) A_{n}(t, X) U_{v}(t, X)=\int_{-\infty}^{+\infty} \int_{0}^{+\infty} v \xi f(t, X, \xi, v) d_{v} d_{\xi}
$$

If the distributions of the molecules are such that molecules maintain on average the same separation distances between each other, in particular the measurable volume between the molecules is always and everywhere the same, then $v$ is a constant and it is seen that $U_{m}(t, X)$ and $U_{v}(t, X)$ coincide. This uniformity situation represents a homogeneous medium, where mass-density is constant throughout. It follows that a difference between these two velocities occurs in a non-homogeneous medium, where variations of mass-density exist.

From the two previous macroscopic velocities, we have two peculiar velocities expressed by,

$$
C=\xi-U_{m}
$$

and

$$
C^{\prime}=\xi-U_{v}
$$

Accounting for macroscopic expansions or compressions of the medium, the proper random motions which are classically associated with diffusive processes are those from which both the macroscopic velocities $U_{m}$ and $U_{v}$ have been subtracted. 
Note that in the above definitions of macroscopic variables, the volume $v$ and the velocity $\xi$ are the basic random variables, with their expected values (or expected values of their functions) being associated to the flow properties. The time $t$ and position $X$ play a different role. Therefore any macroscopic flow property has an assigned microscopic random variable. This is different from the classical description in the Boltzmann equation, where the mass-density has no randomized component or, more precisely, is associated with the "constant random variable", 1.

\section{The Kinetic Equation and Subsequent Set of Hydrodynamic Equations}

To derive a kinetic equation for the distribution $f(t, X, \xi, v)$ some physical assumptions are obviously required. Here we assume that this distribution function is conserved in the new generalized phase space. In addition, changes in the momentum of a given molecule are only important during instantaneous interactions with other molecules. It follows that we may replace the force term in equation (7) and write:

$$
\frac{\partial f}{\partial t}+(\xi \cdot \nabla) f+\left(F_{e x t} \cdot \nabla_{\xi}\right) f+W \frac{\partial f}{\partial v}=I(f, f),
$$

where $I(f, f)$ is a Boltzmann type of collision integral and $W=\delta v / \delta t$. Then a set of hydrodynamic equations may be derived by taking moments of the kinetic equation with respect to the microscopic random variables $v, \xi$ and $\xi^{2}$, and the constant 1 [11]. This set of equations is written

$$
\begin{aligned}
\frac{D A_{n}}{D t} & =-A_{n} \nabla \cdot U_{m}, \\
\frac{D \bar{\rho}}{D t}= & \frac{\bar{\rho}^{2}}{M}\left[\frac{1}{A_{n}} \nabla \cdot\left[A_{n} J_{v}\right]-W\right], \\
A_{n} \frac{D U_{m}}{D t}= & -\nabla \cdot A_{n}\left(P^{\prime}-\frac{1}{\bar{v}^{2}} J_{v} J_{v}\right), \\
A_{n} \frac{D}{D t}\left[\frac{1}{2} U_{m}^{2}+e_{i n}^{\prime}-\frac{1}{2 \bar{v}^{2}} J_{v}^{2}\right]= & -\nabla \cdot A_{n}\left[\left(P^{\prime}-\frac{1}{\bar{v}^{2}} J_{v} J_{v}\right) \cdot U_{m}\right] \\
& -\nabla \cdot A_{n}\left[q^{\prime}+\frac{1}{\bar{v}} P^{\prime} \cdot J_{v}+\frac{1}{\bar{v}}\left(e_{i n}^{\prime}-\frac{1}{\bar{v}^{2}} J_{v}^{2}\right) J_{v}\right] .
\end{aligned}
$$

We have denoted the material derivative $D / D t \equiv \partial / \partial t+U_{m} \cdot \nabla$. Quantities $J_{v}, P^{\prime}$, and $q^{\prime}$ are the fluxes of, respectively, $v, \xi$, and $\xi^{2}$, due to the real randomized component of molecular motion that is $C^{\prime}$. Consequently, they are here associated with diffusive fluxes and may be approximated using conventional phenomenological first order diffusion models:

$$
\begin{aligned}
\frac{M P_{i j}^{\prime}}{\bar{v}} & =p^{\prime} \delta_{i j}-\mu^{\prime}\left(\frac{\partial U_{v_{i}}}{\partial X_{j}}+\frac{\partial U_{v_{j}}}{\partial X_{i}}\right)-\eta^{\prime} \frac{\partial U_{v_{k}}}{\partial X_{k}} \delta_{i j}, \\
\frac{M q^{\prime}}{\bar{v}} & =-\kappa_{h}^{\prime} \nabla T^{\prime}, \\
\frac{J_{v}}{\bar{v}} & =\kappa_{m}\left[\bar{\rho}^{-1} \nabla \bar{\rho}\right],
\end{aligned}
$$

with $\mu^{\prime}$ a dynamic viscosity, $\kappa_{h}^{\prime}$ a heat conductivity, $\eta^{\prime}$ a bulk viscosity, $\kappa_{m}$ the volume or mass diffusion coefficient, all to be determined in this new framework. Any flux density is taken in respect of the real macroscopic unit of volume of gas, $\bar{v}$. As the complete macroscopic motion, from which the momentum flux $P^{\prime}$ is defined as a diffusive flux, is $U_{v}$, the phenomenological law of diffusion used to express $P^{\prime}$ is applied with a gradient taken over $U_{v}$. The internal energy is $e_{i n}^{\prime}$ and the following relations are also derived from the basic definitions of macroscopic flow properties: $\frac{2}{3} \mu^{\prime}+\eta^{\prime}=0, U_{v}=U_{m}+\bar{v}^{-1} J_{v}, M e_{i n}^{\prime}=(3 / 2) k T^{\prime}$ or $p^{\prime}=(2 / 3) \bar{\rho} e_{i n}^{\prime}$, with $p^{\prime}$ the mean pressure and $T^{\prime}$ the temperature.

The difference in this new set of macroscopic equations is mainly due to the volume flux component $J_{v}$, which is also, in fact, a diffusive flux corresponding to the mass-density of the medium, as spatial distributions of the molecules are represented as a random process in our description compared to the conventional description. The same type of flux has been recently claimed by various authors to be of importance in continuum mechanics $[12,13]$. 


\section{DENSITY PROFILES IN A STEADY STATE HEAT TRANSFER PROBLEM}

The prediction of mass-density profiles in a pure heat conduction problem between two parallel plates is a good initial test for the new set of hydrodynamic equations (17) to (20). In [14] a finite-difference analysis of the nonlinear Boltzmann equation for hard-sphere molecules was used to solve this flow configuration and results were compared to experiments. According to [14], "there is a considerable difference between the mass-density distribution by the full Boltzmann equation and the experiments". Meanwhile, with the same configuration, the classical set of NavierStokes hydrodynamic equations do not predict any actual mass-density profile because the continuity equation simply vanishes while pressure is constant and the temperature is linear.

Consider the set of hydrodynamic equations (17) to (20) in a one-dimensional steady state configuration with $U_{m}=0$. The $x$-axis is normal to the plates. The continuity equation (17) vanishes and equation (18) with a sensible phenomenological description of $W$ leads to [11]:

$$
\bar{\rho} \frac{\partial}{\partial x}\left(\frac{\kappa_{m}}{\bar{\rho}^{2}} \frac{\partial \bar{\rho}}{\partial x}\right)+\kappa_{m}\left(\frac{1}{\bar{\rho}} \frac{\partial \bar{\rho}}{\partial x}\right)^{2}=0 .
$$

Neglecting non-linear terms, equation (24) reduces to

$$
\frac{\partial^{2} \bar{\rho}}{\partial x^{2}}=0
$$

which has the solution $\bar{\rho}(x)=C_{t 2}+x C_{t 1}$. Therefore the mass-density profile is linear, and this mainly derives from the diffusive component appearing in the mass-density equation (18) and not from an equation of state. According to experimental data [14] the mass-density profiles are clearly linear.

\section{CONCLUSION}

In this paper, we have argued that conventional derivations of the Boltzmann kinetic equation in dilute gases introduce a definition of macroscopic mass-density of the medium which contradicts macroscopic thermodynamic properties. In particular, the mass-density is associated with a constant microscopic random variable which implies a medium of uniformly distributed molecules. By introducing a proper random variable associated with the spatial distributions of molecules into the microscopic kinetic description, we have shown that the resulting macroscopic hydrodynamic model has a new diffusive component in the mass-density. Early tests on a benchmark heat conduction problem indicate this new model has some utility.

\section{REFERENCES}

1. S. G. Brush, The Kind of Motion We Call Heat: A History of the Kinetic Theory of Gases in the 19 ${ }^{\text {th }}$ Century, vol. 2, North-Holland Personal Library, 1999, $3^{\text {rd }}$ edn.

2. C. Callender, Entropy 6, 11-20 (2004).

3. J. C. Maxwell, Philosophical Transactions of the Royal Society of London 170, 231-256 (1878).

4. J. Binney, and S. Tremaine, Galactic Dynamics, Princeton University Press, 1987, $2^{\text {nd }}$ edn.

5. P. Chavanis, Physica A 359, 177-212 (2006).

6. C. Cercignani, Rarefied Gas Dynamics: From Basic Concepts to Actual Calculations, Cambridge University Press, 1990.

7. C. Cercignani, Theory and Application of the Boltzmann Equation, Scottish Academic Press, 1975.

8. S. Chapman, and T. Cowling, The Mathematical Theory of Non-Uniform Gases, Cambridge Mathematical Library, $1970,3^{\text {rd }}$ edn.

9. J. M. Blatt, and A. H. Opie, Journal of Physics A 7, 1895-1906 (1974).

10. P. Degond, L. Pareschi, and G. Russo, Modeling and Computational Methods for Kinetic Equations, Birkhäuser, 2004.

11. S. K. Dadzie, J. M. Reese, and C. R. McInnes, Physica A 387, 6079-6094 (2008).

12. H. Brenner, Physica A 349, 60-132 (2005).

13. B. C. Eu, Journal of Chemical Physics 129, 094502 (2008).

14. T. Ohwada, Physics of Fluids 8, 2153-2160 (1996). 